\section{The size of the cervix and its relationship with age and parity}

\section{Marie-Andrée Harvey \\ Division of Urogynecology and \\ Reconstructive Surgery, Departments of \\ Obstetrics and Gynecology and of \\ Urology, Queen's University, Kingston \\ (ON), Canada}

\begin{abstract}
The aim of this study was to determine whether cervical diameter changes with age, parity or hormonal status, as we postulate that the cervical size can influence pelvic organ prolapse quantification (POPQ) staging, as apical stage 0 assumes a cervical diameter of $\leq 2 \mathrm{~cm}$. We reviewed all hysterectomies performed at a single university-affiliated hospital and compared ex vivo cervical diameter based on parity, menopausal status and hormonal exposure. Specimen results were available for 127 women, mean age 48 years (rage 24-89), half were parous. Most (83\%) surgical indications were benign. Of the 77 women whose menopausal status was known, 25 were postmenopausal. The mean cervical diameter was greater in women $<55$ years $[3.5 \mathrm{~cm}, 95 \%$ confidence interval (CI) (3.4-3.6) vs $2.8 \mathrm{~cm} \mathrm{95 \% CI}$ (2.7-2.9)]. Parity was also associated with greater cervical diameter $[3.4 \mathrm{~cm}, 95 \% \mathrm{CI}(3.2-$

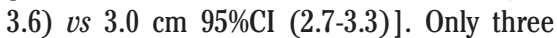
women had a cervix $\leq 2 \mathrm{~cm}$. The cervical diameter is considerably larger in younger and parous women. Consequently, its size may, conceptually, affect the apical staging of $\mathrm{POPQ}$. Such misclassification could obscure identification and affect interpretation of studies evaluating the natural history or outcomes following surgical treatment of prolapse.
\end{abstract}

\section{Introduction}

Since its introduction, the pelvic organ prolapse quantification (POPQ) system $^{1}$ has become the standard assessment tool for prolapse evaluation in both clinical practice and research. While the POPQ system has been shown to be reliable, ${ }^{2}$ no anatomical validation study is available.

One area of controversy surrounds the distinction between stage 0 and stage 1 of the POPQ system. While it is generally agreed that POPQ stage 1 is asymptomatic, ${ }^{3,4}$ it is unknown whether stage 1 represents normal support or early but subclinical prolapse. Studies have identified a high prevalence of stage 1 pelvic organ support in populations unlikely to exhibit prolapse: $50 \%$ of women aged $18-29$ years, ${ }^{5}$ $58 \%$ of nullipara of any age, ${ }^{6}$ and $43-66 \%$ of primigravida in their second trimester. ${ }^{5,7}$ The correct classification of otherwise asymptomatic women as stage 0 and stage 1 is important not only for the accurate diagnosis and prognosis of individual patients, but also to better understand the natural history of prolapse. ${ }^{8}$

Apical staging is based on the distance between the cervical leading edge (Point $\mathrm{C}$ ) and the hymen during Valsalva. Stage I is defined as Point $\mathrm{C}$ being more than one $\mathrm{cm}$ above the hymen and $2 \mathrm{~cm}$ or more shorter than the total vaginal length (TVL) (i.e., $-1<$ Point C $\leq-$ [TVL $-2 \mathrm{~cm}]$ ). Since the cervix is naturally perpendicular to the long axis of the vagina when not held by the speculum (Figure 1), the bulk of the cervix may affect the location of Point $\mathrm{C}$ (Figure 2A and B). Specifically, a woman whose cervix exceeded $2 \mathrm{~cm}$ in diameter could never be classified as stage 0 (i.e., >TVL-2 cm), even in the absence of prolapse.

Surprisingly, there are few data on the size and variability of cervical diameter. Because the POPQ system was originally developed in a cohort of older women (mean age 61 years), the effects of age or parity on cervix size may not have been appreciated. The goal of our study was to characterize the size of the cervix based on age, parity and menopausal status.

\section{Materials and Methods}

We identified all hysterectomy specimens via electronic search of the hospital pathology database, from a two-year period in a 450-bed tertiary care teaching hospital. A single experienced pathology technician, unaware of the study, measured each specimen to the nearest millimeter using a ruler. While the sagittal (front-to-back) and coronal (right-to-left) plane diameters were measured, only the larger dimension was recorded, and is referred to as cervical diameter throughout. The cervical length was measured from the external to the internal os after sectioning. Cases were eligible if cervical measurements, age and final pathology were available. The Queen's University Research Ethics Board approved this study and waived consent.

A blinded research assistant performed a standardized review of the hospital and private office charts to obtain age, parity and hormonal exposure at time of hysterectomy. Hormonal exposure was categorized as endogenous (premenopausal), exogenous (post-menopausal using any estrogen therapy) or none (postmenopausal, having not used hormonal replacement for $>6$ months).
Correspondence: Marie-Andrée Harvey,

Department of Obstetrics and Gynecology, 76 Stuart Street, Kingston, Ontario K7L 4P8 Canada. Tel.: +1.613.548.6115 - Fax: +1.613.548.1330

E-mail: harveym@queensu.ca

Key words: Anatomy and histology; comparative; cervix uteri; pelvic organ prolapse; uterine prolapse; pelvic organ prolapse quantification.

Acknowledgements: the author would like to thank Mrs. Wilma Hopman (Kingston General Hospital) for the statistical analysis and Mrs. Robin MacNeil for the artwork.

Conflict of interest: the author declares no potential conflict of interest.

Received for publication: 26 May 2015

Accepted for publication: 1 July 2016.

This work is licensed under a Creative Commons Attribution NonCommercial 4.0 License (CC BYNC 4.0).

(C) Copyright M-A. Harvey, 2016

Licensee PAGEPress, Italy

Urogynaecologia 2016; 29:171

doi:10.4081/uij.2016.171

Cervical diameter and length were tested for normality (skewness, kurtosis, Shapiro-Wilk's W), and correlated with age using Pearson's or Spearman's correlation coefficients as appropriate. Cervix size was then compared analysis of variance between 10-year age strata $(<35$, $35-44,45-54,55-64$, and $\geq 65$ years) and differences identified (Tukey's post hoc test). Univariate analyses (Student's $t$ test) compared the cervical diameter and length by parity (yes/no) and hormonal exposure (exo/endogenous versus none). Post hoc sensitivity analysis was performed by excluding subjects with a condition that might increase cervical size (cervical polyp, cervical fibroids, and cervical cancer).

\section{Results}

During January 2002 and December 2003, 145 hysterectomy specimens were processed of which 127 met inclusion criteria. The population sampled was typically middle-aged and of those for whom the hormonal exposure status was available $(n=88)$, half were premenopausal (Table 1). Of the 26 postmenopausal women, four were receiving exogenous estrogens. The final pathology identified mostly benign conditions (83\%). Only eight women had a condition that might cause cervical enlargement: cervical fibroids ( $1 \mathrm{~cm}$ and $6 \mathrm{~cm}, \mathrm{n}=2$ ), cervical polyps (5 and 8 
$\mathrm{mm}, \mathrm{n}=4)$ and cervical cancer $(\mathrm{n}=2)$.

The mean cervical diameter was $3.3 \mathrm{~cm}$ [95\% confidence interval $(\mathrm{CI})(3.2-3.5 \mathrm{~cm})]$, normally distributed, and correlated inversely with age (Pearson's $r=-0.40, \mathrm{P}<0.001$ ) (Figure 3 ). As shown in Figure 4 and confirmed by Tukey's post hoc test, a breakpoint exists around 55 years of age. Specifically, $82 \%$ (79/96) of women younger than 55 years had a cervix $\geq 3 \mathrm{~cm}$ in diameter [mean $3.5 \mathrm{~cm} 95 \% \mathrm{CI}$ $(3.4-3.6 \mathrm{~cm})]$, versus only $42 \%(13 / 31)$ of women 55 and older [mean $2.8 \mathrm{~cm}, 95 \%$ (CI $2.7-2.9 \mathrm{~cm}$ ); pooled t-test for equal variance $\mathrm{P}<0.001$ ]. Only three women (age 53, 61, and 73 year) had a cervix $\leq 2 \mathrm{~cm}$ in diameter. Furthermore, the cervix was on average $4 \mathrm{~mm}$ wider in parous women, and in women with either endogenous or exogenous hormonal exposure (Table 2).

The mean cervical length was $2.8 \mathrm{~cm}$ [ $95 \%$ CI $(2.7-2.9 \mathrm{~cm})]$, and not normally distributed. Six women had a cervical length $\leq 2 \mathrm{~cm}$ : of these, two women were less than 45 year-old. While a weak inverse correlation was noted between age and cervical length (Spearman's $\mathrm{r}=-0.21, \mathrm{P}<0.02$ ), no significant difference was found when subjects were classified by decade (Kruskal-Wallis $\chi^{2}$ test: $7.8, \mathrm{P}=0.12$ ).

The sensitivity analysis excluding women with a condition potentially increasing cervical size did not affect these results appreciably. Hormonal status was highly correlated with age and the independent effects of each variable could not be distinguished.

\section{Discussion}

We identified moderate but clinically and statistically important anatomical differences

Table 1. Baseline characteristics of the study population (percentages have been rounded up).

\begin{tabular}{lc}
$\begin{array}{l}\text { Age }(\mathrm{n}=127) \\
\text { (mean, SD) }\end{array}$ & $48(+/-12)$ years \\
Surgical pathology (n, \%) & \\
Benign & $105(83 \%)$ \\
Cancerous & $22(18 \%)$ \\
$\quad$ Uterus & $15(12 \%)$ \\
Cervix & $2(2 \%)$ \\
$\quad$ Ovary and other & $5(4 \%)$ \\
Hormonal exposure (n, \%) & \\
Endogenous & $62(49 \%)$ \\
Exogenous & $4(3 \%)$ \\
None & $22(17 \%)$ \\
Not reported & $39(31 \%)$ \\
\hline Parity (n, \%) & \\
Nulliparous & $16(13 \%)$ \\
Parous & $68(54 \%)$ \\
Not reported & $43(34 \%)$ \\
\hline SD, standard deviation. &
\end{tabular}

in cervical size with age, parity and hormonal status. These differences may impact the signification of stage I apical support.

These differences are large enough to potentially misclassify pelvic floor support staging using the POPQ system. The cervical diameter of most young and many older women exceeds $3 \mathrm{~cm}$, a full centimeter longer than the $2 \mathrm{~cm}$ cut point used by POPQ to determine apical stage 0 . Because the cervix and vagina are oriented at roughly right angles to each other, Point $\mathrm{C}$ can only infrequently lie within $2 \mathrm{~cm}$ of TVL and thus be classified as stage 0 . Hence a woman with a cervical diameter larger than $2 \mathrm{~cm}$ will be de facto classified as having stage 1 support even if she has no support defect. This issue is more frequent, but not limited to women who are young, parous or premenopausal. Our findings are congruent with recent findings by Dietz and Mann. ${ }^{9}$ In a study evaluating 764 women, they identified cutoffs (using receiver operator curve) for point $\mathrm{C}$ for clinically symptomatic prolapse. They excluded those women with a dominant prolapse in a compartment other than apical, identifying 363 women available for assessment. They found that stage I, considered by the International Continence Society (ICS) as normal, was in fact likely abnormal as associated with prolapse symptoms. According to the POPQ system, Point $\mathrm{C}$ is the point that represents either the most distal edge of the cervix... The 2-cm buffer around the TVL was chosen empirically in an effort to compensate for vaginal distensibility and the inherent imprecision of the measurement of total vaginal length. ${ }^{1}$ To measure the most distal edge of the cervix in its normal position, the speculum needs to free the cervix to prevent obstructing its movement during Valsalva (Figure 1). It has been our experience that the anterior lip of the cervix is the leading edge

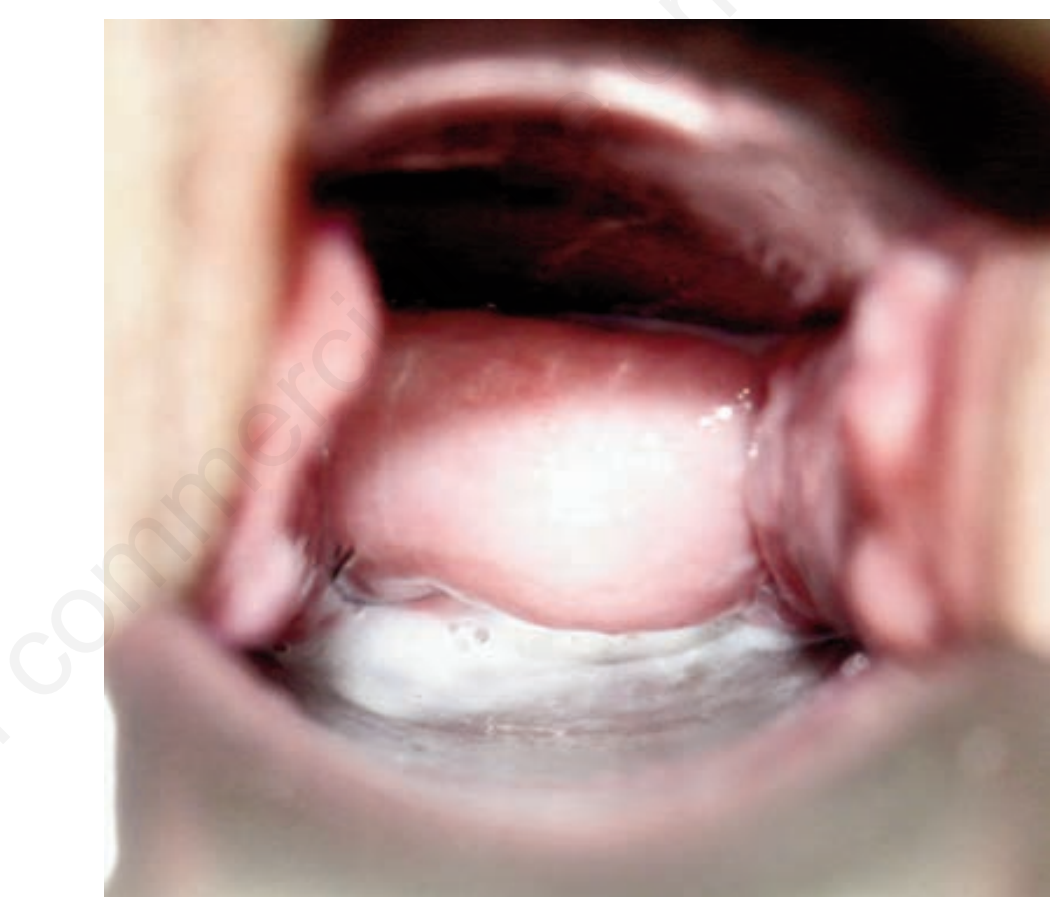

Figure 1. Cervical and vaginal axis orientation. We can see that the leading edge of the cervix is the anterior lip, and that the cervix fills the proximal segment of the anterior vaginal wall. String from an intra uterine device is visible on the patient's right side.

Table 2. Effect of parity and hormonal exposure on mean cervical diameter and length, in $\mathbf{c m}$.

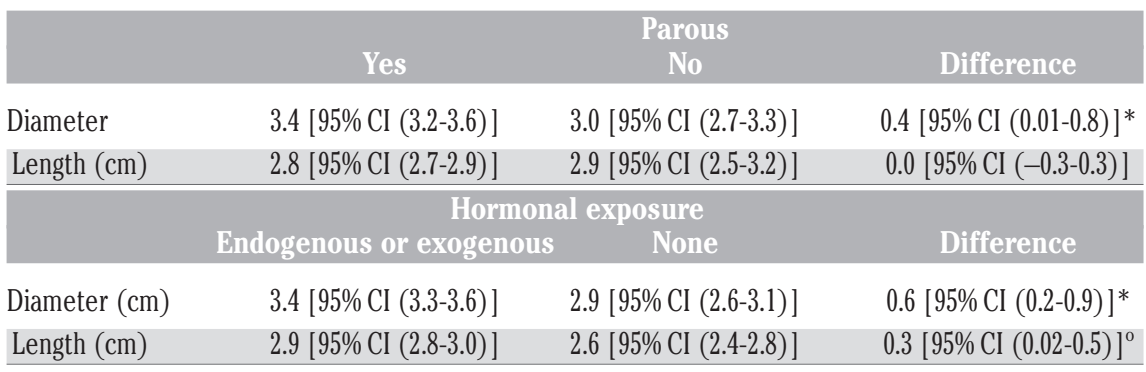

$\mathrm{CI}$, confidence interval. *Statistically significant using $t$-test; ${ }^{\circ}$ statistically significant using Kruskal-Wallis test. 
even in the women with retroverted uterus. This observation, also illustrated in the Standardization publication, ${ }^{1}$ suggests that the sagittal diameter of the cervix determines Point $\mathrm{C}$, and thus affects the staging of apical support. The cervix of a younger woman (Figure 2), being larger, fills the vaginal apex and places its leading edge closer to the hymen than that of an older woman (Figure 3). Consequently the distance between the leading edge of the cervix and the hymen will necessarily be classified as apical support stage 1 , even in the absence of any descent or defective support. Cervical length was not influenced by parity, age decades or hormonal status in this study. The length of the cervix might have an impact on staging and this is assumed to be demonstrated on POPQ by the determination of Point $\mathrm{D}$ in relation to Point C: a well-supported point $\mathrm{D}$ and a low Point $\mathrm{C}$ may represent a long cervix. Berger et al. found that total cervical length as obtained on magnetic resonance imaging (MRI) was associated with apical descent in women with stage II or greater anterior wall prolapse. ${ }^{10}$ In support of this association between cervical length and prolapse, Berger et al. correlated both cervical length and difference between the measurements of point C and D with POPQ stage and found that women with prolapse (as defined as at least anterior wall reaching $1 \mathrm{~cm}$ outside of hymen) had longer cervix greater distance between point $\mathrm{C}$ and D. ${ }^{10}$ However, this study had one major limitation: the cervical length was the total length measured on MRI, from the external to the internal os, which does not reflect the relationship between length of the vaginal portio, which arguably is the relevant one when support is assessed on physical examination during POPQ measurements. One can see that to reach Point $C$ from Point $D$, one not only has to come down the length of the exocervix from $\mathrm{D}$ to the edge of the posterior cervix, but then across the cervical width to the edge of the anterior cervix, where is located
A

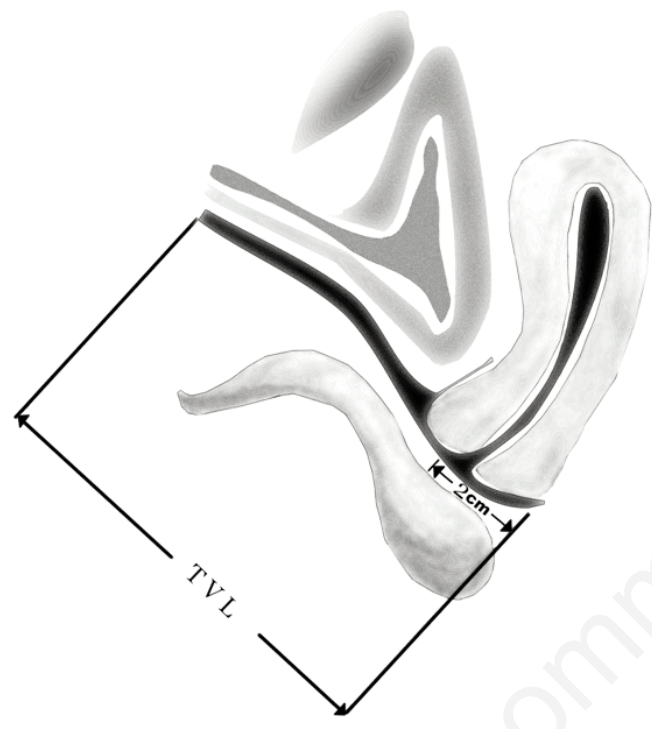

B

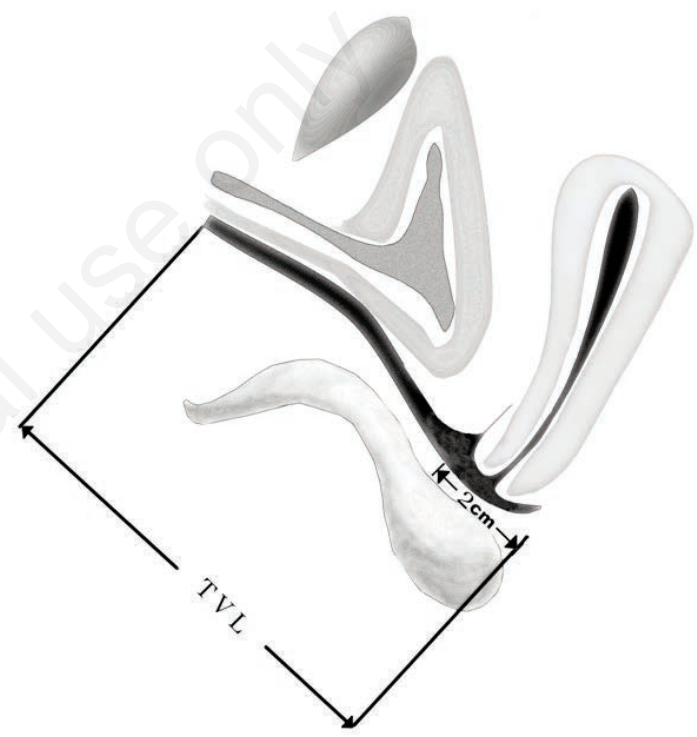

Figure 2. Sagittal section of the vagina showing apical support stage 1, according to pelvic organ prolapse quantification due to larger (A) and smaller (B) cervix (original artwork courtesy by Robin MacNeil).

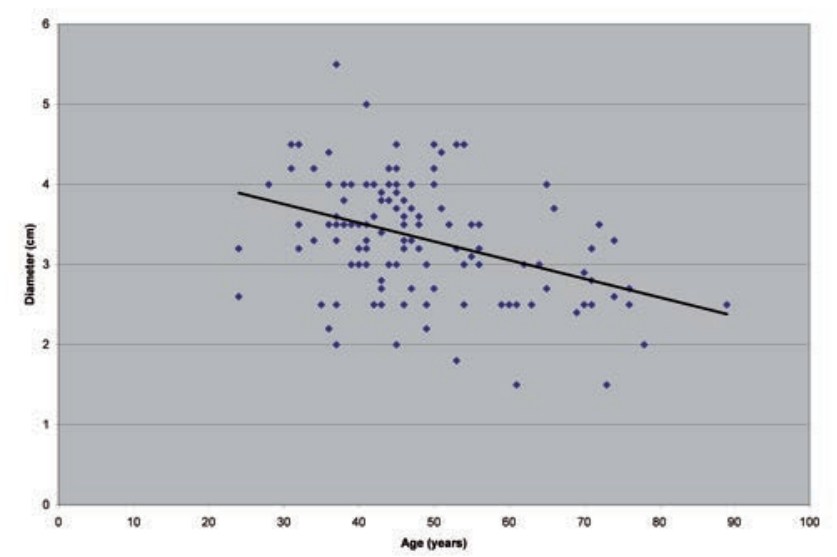

Figure 3. Scatter plot showing raw data showing the relationship between age and cervical diameter.

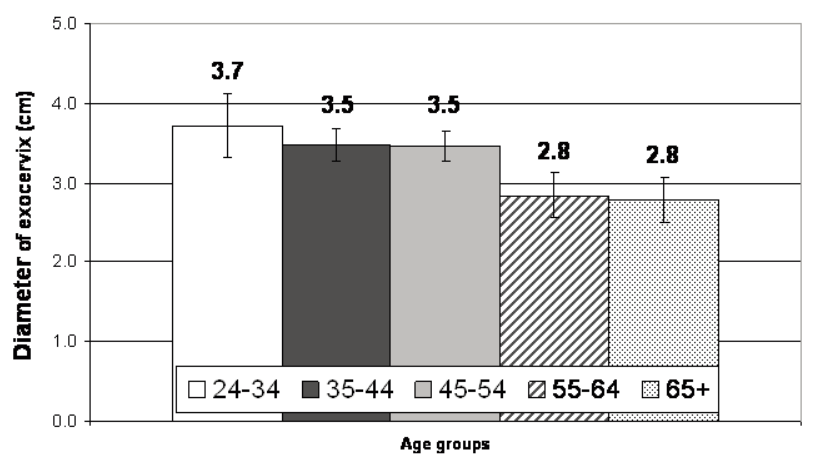

Figure 4. Diagram of cervical diameter, by 10 -years age strata. 
Point $\mathrm{C}$, illustrating the impact of a large cervix on the determination of staging.

One prior small study investigated the influence of hormonal state on cervical size, ${ }^{11}$ which was congruent with ours. Bartoli et al. used MRI to assess the uterus of 16 volunteers: eight nulligravida on cyclical contraception, six women naturally cycling (one of them parous), and two nulliparous postmenopausal women (one on estrogen replacement). Postmenopausal women had a narrower cervix diameter (mean $2 \mathrm{~cm}$, standard deviation 0 $\mathrm{cm}$ ) than their premenopausal counterparts (mean $2.7 \mathrm{~cm}$, standard deviation $0.4 \mathrm{~cm}$ ), independent of hormonal contraception or timing in natural cycle. While the difference of nearly $1 \mathrm{~cm}$ between older and younger women was similar to our findings, the actual size of cervix in younger women was narrower than in our study, possibly due to differences in approaches (ex vivo versus in vivo), smaller sample size and low parity.

Misclassification occurs when normal patients are deemed to have disease present or vice versa. Of course, one could simply deem that both stage 0 and 1 be classified as normal, especially given that stage 1 is thought by most, but not all, ${ }^{9}$ to be asymptomatic. Both mild and subclinical degrees of pelvic organ descent should not be dismissed until we better understand the natural history and pathophysiology of pelvic organ prolapse. In fact, when the anterior vaginal wall reaches the hymen, most Point $\mathrm{C}$ can be found at $-4.5 \mathrm{~cm} .^{12}$ Such stage I support, while considered normal by most, is clearly associated with an abnormal overall support. It is thought that nearly $60 \%$ of the anterior wall support is provided by the apex..$^{13}$ Differentiating early mild prolapse from normal support is important to advancing this understanding. An analogy can be made with low-grade squamous epithelial lesion, which is not classified as normal, as it is considered a pre-clinical lesion that carries a risk (albeit small) of progressing into a clinically significant condition. Clearly, a woman who has a TVL of $8 \mathrm{~cm}$ and is classified as stage I based on a point $C$ measured at $-2 \mathrm{~cm}$ is not the same as another where point $\mathrm{C}$ is obtained at $-5 \mathrm{~cm}$. Our findings suggest that the arbitrary cutoff of TVL $-2 \mathrm{~cm}$ may not be valid in most women based on measurements on anatomical specimens.

We acknowledge some important limitations. The independent effects of age and of hormone status could not be distinguished as too few older women were on estrogen replacement therapy and too few young women were estrogen deficient. Our sample size was too small to allow multiple group comparison or multivariate analyses. We could not distinguish between sagittal and coronal diameter as only the greater measurement was recorded on the pathology report. Most importantly, there was no preoperative evaluation of prolapse to correlate pathological finding with staging of support.

\section{Conclusions}

The cervix is considerably larger in diameter in younger and parous women. By POPQ design, cervix size may affect the apical staging of POPQ, staging a woman as stage I when there is no descent. Such misclassification could obscure identification and affect the interpretation of studies evaluating the natural history or surgical treatment of prolapse.

\section{References}

1. Bump RC, Mattiasson A, Bo K, et al. The standardization of terminology of female pelvic organ prolapse and pelvic floor dysfunction. Am J Obstet Gynecol 1996; 175:10-7.

2. Hall AF, Theofrastous JP, Cundiff GW, et al. Interobserver and intraobserver reliability of the proposed international continence society, society of gynecologic surgeons, and american urogynecologic society pelvic organ prolapse classification system. Am J Obstet Gynecol 1996;175:1467-71.

3. Swift SE, Tate SB, Nicholas J. Correlation of symptoms with degree of pelvic organ support in a general population of women: what is pelvic organ prolapse? Am J Obstet Gynecol 2003;189:372-7.

4. Jelovsek JE, Maher C, Barber MD. Pelvic organ prolapse. Lancet 2007;369:1027-38.

5. O'Boyle AL, Woodman PJ, O'Boyle JD, et al. Pelvic organ support in nulliparous pregnant and nonpregnant women: a case control study. Am J Obstet Gynecol 2002;187: 99-102.

6. Swift SE. The distribution of pelvic organ support in a population of female subjects seen for routine gynecologic health care. Am J Obstet Gynecol 2000;183:277-85.

7. Harvey MA, Eason E, Wells GA. Genital prolapse following childbirth: a study of its incidence and determinants. Int Urogynecol J 2006;17:S367-S.

8. Wiegersma M, Panman CM, Lisman-Van Leeuwen Y, Dekker JH. More research is needed before we regard pop-q stage 1 prolapse as normal. Int Urogynecol J 2014;25:1291.

9. Dietz HP, Mann KP. What is clinically relevant prolapse? An attempt at defining cutoffs for the clinical assessment of pelvic organ descent. Int Urogynecol J 2014; 25:451-5.

10. Berger MB, Ramanah R, Guire KE, Delancey JO. Is cervical elongation associated with pelvic organ prolapse? Int Urogynecol J 2012;23:1095-103.

11. Bartoli JM, Moulin G, Delannoy L, et al. The normal uterus on magnetic resonance imaging and variations associated with the hormonal state. Surg Radiol Anat 1991;13:213-20.

12. Rooney K, Kenton K, Mueller ER, et al. Advanced anterior vaginal wall prolapse is highly correlated with apical prolapse. Am J Obstet Gynecol 2006;195:1837-40.

13. Summers A, Winkel LA, Hussain HK, DeLancey J0. The relationship between anterior and apical compartment support. Am J Obstet Gynecol 2006;194:1438-43. 